MKPH-T-03-7

\title{
Electromagnetic form factors of the nucleon in relativistic baryon chiral perturbation theory
}

\author{
Thomas Fuchs, ${ }^{1}$ Jambul Gegelia,, ,, * and Stefan Scherer ${ }^{1}$ \\ ${ }^{1}$ Institut für Kernphysik, Johannes Gutenberg-Universität, D-55099 Mainz, Germany \\ ${ }^{2}$ High Energy Physics Institute, Tbilisi State University, \\ University St. 9, 380086 Tbilisi, Georgia
}

(Dated: May 23, 2003)

\begin{abstract}
The electromagnetic form factors of the nucleon are calculated at order $q^{4}$ in the relativistic formulation of baryon chiral perturbation theory. In order to obtain a consistent power counting for the renormalized diagrams we make use of the extended on-mass-shell renormalization scheme which we have discussed in a recent paper. We analyze the Dirac and Pauli form factors, $F_{1}$ and $F_{2}$, as well as the electric and magnetic Sachs form factors, $G_{E}$ and $G_{M}$. Our results are compared with those obtained in the heavy-baryon approach and in the (relativistic) infrared regularization. In addition, the Sachs form factors are compared with experimental data. We confirm previous findings that a one-loop calculation of the electromagnetic form factors does not generate sufficient curvature for the Sachs form factors $G_{E}^{p}, G_{M}^{p}$, and $G_{M}^{n}$. Moreover, the electric form factor of the neutron is very sensitive to higher-order contributions.
\end{abstract}

PACS numbers: 12.39.Fe, 13.40.Gp

\footnotetext{
${ }^{*}$ Alexander von Humboldt Research Fellow
} 


\section{INTRODUCTION}

Chiral perturbation theory $(\chi \mathrm{PT})$ for mesons [1, 2] is the effective field theory of lowenergy QCD in the vacuum sector (baryon number zero), expressed in terms of the effective degrees of freedom active at low energies. These are the Goldstone bosons resulting from the spontaneous symmetry breaking of the chiral group $\mathrm{SU}(N)_{L} \times \mathrm{SU}(N)_{R}$, reflecting the symmetry of the QCD Lagrangian for $N$ massless quark flavors, down to its vectorial subgroup $\mathrm{SU}(N)_{V}$, denoting the symmetry of the vacuum. The effective Lagrangian is organized in terms of a simultaneous expansion in powers of (covariant) derivatives and quark-mass terms, and a perturbative expansion of observables is expected to work for momenta sufficiently small in comparison with the scale of chiral symmetry breaking, $\Lambda_{\chi} \sim 1 \mathrm{GeV}$. Perturbative calculations in the mesonic sector are feasible due to the existence of a consistent power counting scheme [1, 2] establishing a connection between the chiral expansion and the loop expansion (see, e.g., Ref. [3] for a recent overview).

The one-nucleon sector (baryon number one) turns out to be more complicated [4] because of the presence of a new mass scale - the mass of the nucleon - which does not vanish in the chiral limit. In particular, loop diagrams containing internal nucleon lines may contribute to low orders in the chiral expansion. Therefore, the relation between the chiral expansion and the loop expansion appears to be lost [4]. This problem has been solved in the framework of heavy-baryon chiral perturbation theory $(\mathrm{HB} \chi \mathrm{PT})[5$, 6], where the power counting is restored in terms of an additional $1 / m$ expansion, and perturbative calculations are again possible. However, $\mathrm{HB} \chi \mathrm{PT}$ has its own shortcomings: the corresponding perturbation series fails to converge in part of the low-energy region [7]. Moreover, at higher orders in the chiral expansion, the expressions due to $1 / m$ corrections of the Lagrangian become increasingly complicated [8, 9]. These disadvantages are related to the nonrelativistic momentum expansion in this approach.

Recently, various methods have been proposed to establish a consistent power counting within a relativistic approach [10, 11, 12, 13, 14, 15, 16, 17]. In this work, we will discuss the electromagnetic form factors of the nucleon within the extended on-mass-shell (EOMS) renormalization scheme of Ref. [17]. In this scheme renormalization is performed by expanding a given Feynman diagram in terms of small quantities and subtracting those terms which violate the power counting. Since the subtracted terms are regular in the small quantities, they can be absorbed in a finite number of low-energy constants of the chiral Lagrangian. So far, the new method has been applied to a discussion of the mass and the scalar form factor of the nucleon at $\mathcal{O}\left(q^{4}\right)$ [17].

The electromagnetic form factors parameterize the single-nucleon matrix element of the electromagnetic current operator and thus reflect the electromagnetic structure of the nucleon. The matrix element is usually described in terms of either the Dirac and Pauli form factors, $F_{1}$ and $F_{2}$, or the electric and magnetic Sachs form factors, $G_{E}$ and $G_{M}$. The latter are popular, because their Fourier transforms in the Breit frame come closest to an interpretation as the distribution of charge and magnetization inside the nucleon [18, 19] (for a recent discussion and an overview of the existing relevant form factor measurements, see Refs. [20, 21]). For low momentum transfers, $Q^{2} \leq 1 \mathrm{GeV}^{2}$, the Sachs form factors $G_{E}^{p}, G_{M}^{p}$, and $G_{M}^{n}$ are reasonably well described by a dipole form factor $G_{D}$,

$$
G_{E}^{p} \approx \frac{G_{M}^{p}}{\mu_{p}} \approx \frac{G_{M}^{n}}{\mu_{n}} \approx G_{D},
$$


where $G_{D}\left(Q^{2}\right)=\left[1+Q^{2} /\left(0.71 \mathrm{GeV}^{2}\right)\right]^{-2}$. As has been stressed in Ref. [21], there is essentially no physical motivation for such a form and it is also not able to describe "fine structure" in the data. The electric form factor of the neutron, $G_{E}^{n}$, is the least precisely known due to the lack of a free neutron target and the fact that it is small. However, recent polarization experiments have improved our empirical knowledge of $G_{E}^{n}$ considerably (see Ref. [21] and references therein). Clearly, a quantitative description of these four form factors is a stringent test for any theory or model of the strong interactions (see, e.g., Refs. $22,23,24,25,26,27,28,29,30])$. In chiral perturbation theory, these form factors have been calculated within the early relativistic approach [4], $\mathrm{HB} \chi \mathrm{PT}$ [6, 31], the small-scale expansion [32], and within the relativistic infrared regularization approach 33].

Our work is organized as follows. In Sec. II we shortly review the effective Lagrangian and list the interaction terms relevant for our calculation. In Sec. III the Dirac and Pauli as well as the Sachs form factors are discussed. Our results are compared with the $\mathrm{HB} \chi \mathrm{PT}$ calculation, the method of infrared regularization, and experimental data. A summary and some concluding remarks are given in Sec. IV] Some technical details can be found in the appendices.

\section{CHIRAL LAGRANGIAN}

The relevant effective Lagrangian contains two pieces,

$$
\mathcal{L}_{\text {eff }}=\mathcal{L}_{\pi}+\mathcal{L}_{\pi N}
$$

where the first part is the pure mesonic Lagrangian and the second part consists of terms which are bilinear in the isospin doublet $\Psi$ containing the proton and neutron fields. The different terms of the effective Lagrangian can be organized according to the chiral derivative and quark-mass expansion [1, 2, 4],

$$
\mathcal{L}_{\text {eff }}=\mathcal{L}_{2}+\mathcal{L}_{\pi N}^{(1)}+\mathcal{L}_{\pi N}^{(2)}+\mathcal{L}_{\pi N}^{(3)}+\mathcal{L}_{\pi N}^{(4)}+\cdots
$$

where the subscripts (superscripts) in $\mathcal{L}_{\pi}\left(\mathcal{L}_{\pi N}\right)$ refer to the order in the expansion, and the ellipsis denotes terms of higher order which are not relevant for our calculation.

From the mesonic sector we only need the lowest-order Lagrangian $\left[\mathcal{O}\left(q^{2}\right)\right]$ including the quark-mass term and the coupling to an external electromagnetic field $\mathcal{A}^{\mu}$ in terms of the isovector vector field $v^{\mu}=-e \frac{\tau_{3}}{2} \mathcal{A}^{\mu}\left(e^{2} / 4 \pi \approx 1 / 137, e>0\right)[2],{ }^{1}$

$$
\mathcal{L}_{2}=\frac{F^{2}}{4} \operatorname{Tr}\left(\partial_{\mu} U \partial^{\mu} U^{\dagger}\right)+\frac{F^{2} M^{2}}{4} \operatorname{Tr}\left(U^{\dagger}+U\right)+i \frac{F^{2}}{2} \operatorname{Tr}\left[\left(\partial_{\mu} U U^{\dagger}+\partial_{\mu} U^{\dagger} U\right) v^{\mu}\right]+\cdots
$$

Here, $F$ denotes the pion decay constant in the chiral limit, $F_{\pi}=F[1+\mathcal{O}(\hat{m})]=92.4$ $\mathrm{MeV}$, and $M^{2}=2 B \hat{m}$ is the lowest-order prediction for the pion mass squared, where $B$ is related to the quark condensate $\langle\bar{q} q\rangle_{0}$ in the chiral limit [2, 35]. We make use of the isospin-symmetric limit $m_{u}=m_{d}=\hat{m}$. The triplet of pion fields is collected in the matrix

\footnotetext{
${ }^{1}$ In the mesonic sector, the isoscalar coupling to the external electromagnetic field only becomes relevant at $\mathcal{O}\left(q^{6}\right)$ [34].
} 
$U$

$$
\begin{aligned}
U(x) & =u^{2}(x)=\exp \left(\frac{i \Phi(x)}{F}\right), \\
\Phi & =\vec{\tau} \cdot \vec{\phi}=\left(\begin{array}{cc}
\pi^{0} & \sqrt{2} \pi^{+} \\
\sqrt{2} \pi^{-} & -\pi^{0}
\end{array}\right) .
\end{aligned}
$$

The lowest-order pion-nucleon Lagrangian is given by [4]

$$
\mathcal{L}_{\pi N}^{(1)}=\bar{\Psi}\left(i \not D-m+\frac{\stackrel{\circ}{g}_{A}}{2} \gamma^{\mu} \gamma_{5} u_{\mu}\right) \Psi
$$

with

$$
\begin{aligned}
D_{\mu} \Psi & =\left(\partial_{\mu}+\Gamma_{\mu}-i v_{\mu}^{(s)}\right) \Psi, \\
\Gamma_{\mu} & =\frac{1}{2}\left[u^{\dagger} \partial_{\mu} u+u \partial_{\mu} u^{\dagger}-i\left(u^{\dagger} v_{\mu} u+u v_{\mu} u^{\dagger}\right)\right], \\
u_{\mu} & =i\left[u^{\dagger} \partial_{\mu} u-u \partial_{\mu} u^{\dagger}-i\left(u^{\dagger} v_{\mu} u-u v_{\mu} u^{\dagger}\right)\right] .
\end{aligned}
$$

In Eq. (3),$m$ and $\stackrel{\circ}{g}_{A}$ refer to the chiral limit (at fixed strange-quark mass) of the physical nucleon mass and the axial-vector coupling constant, respectively. In the definition of the covariant derivative we follow Ref. [8], where $\Gamma_{\mu}$ only contains traceless external fields and the coupling to the isosinglet vector field $v_{\mu}^{(s)}$ is considered separately.

The next-to-leading-order pion-nucleon Lagrangian contains seven low-energy constants $c_{i}$ [4, 9], where only the terms proportional to $c_{1}, c_{2}, c_{4}, c_{6}$, and $c_{7}$ contribute to the calculation of the electromagnetic form factors at $\mathcal{O}\left(q^{4}\right)$,

$$
\begin{aligned}
\mathcal{L}_{\pi N}^{(2)}= & c_{1} \operatorname{Tr}\left(\chi_{+}\right) \bar{\Psi} \Psi-\frac{c_{2}}{4 m^{2}}\left[\bar{\Psi} \operatorname{Tr}\left(u_{\mu} u_{\nu}\right) D^{\mu} D^{\nu} \Psi+\text { h.c. }\right] \\
& +\bar{\Psi}\left[i \frac{c_{4}}{4}\left[u_{\mu}, u_{\nu}\right]+\frac{c_{6}}{2} f_{\mu \nu}^{+}+\frac{c_{7}}{2} v_{\mu \nu}^{(s)}\right] \sigma^{\mu \nu} \Psi+\cdots,
\end{aligned}
$$

where h.c. refers to the Hermitian conjugate and

$$
\begin{aligned}
\chi_{+} & =M^{2}\left(U+U^{\dagger}\right), \\
v_{\mu \nu}^{(s)} & =\partial_{\mu} v_{\nu}^{(s)}-\partial_{\nu} v_{\mu}^{(s)}, \\
f_{\mu \nu}^{ \pm} & =u f_{\mu \nu}^{L} u^{\dagger} \pm u^{\dagger} f_{\mu \nu}^{R} u, \\
f_{\mu \nu}^{L} & =\partial_{\mu} l_{\nu}-\partial_{\nu} l_{\mu}-i\left[l_{\mu}, l_{\nu}\right], \\
f_{\mu \nu}^{R} & =\partial_{\mu} r_{\nu}-\partial_{\nu} r_{\mu}-i\left[r_{\mu}, r_{\nu}\right],
\end{aligned}
$$

with $r_{\mu}=l_{\mu}=-e \tau_{3} \mathcal{A}_{\mu} / 2$ and $v_{\mu}^{(s)}=-e \mathcal{A}_{\mu} / 2$. With the convention of choosing $f_{\mu \nu}^{+}$to be traceless, $c_{6}\left(c_{7}\right)$ will be related to the isovector (isoscalar) magnetic moment of the nucleon in the chiral limit.

The complete Lagrangians at third and fourth order can be found in Refs. [8, 9] and [9], respectively. Again, we only list the terms needed for the calculation of the electromagnetic 
form factors, ${ }^{2}$

$$
\begin{aligned}
\mathcal{L}_{\pi N}^{(3)}= & \frac{i}{2 m} d_{6} \bar{\Psi}\left[D^{\mu}, f_{\mu \nu}^{+}\right] D^{\nu} \Psi+\text { h.c. } \\
& +\frac{2 i}{m} d_{7} \bar{\Psi}\left(\partial^{\mu} v_{\mu \nu}^{(s)}\right) D^{\nu} \Psi+\text { h.c. }+\cdots, \\
\mathcal{L}_{\pi N}^{(4)}= & \bar{\Psi}\left[-2 e_{54}\left(\partial^{\lambda} \partial_{\lambda} v_{\mu \nu}^{(s)}\right)-\frac{1}{2} e_{74}\left[D^{\lambda},\left[D_{\lambda}, f_{\mu \nu}^{+}\right]\right]\right. \\
& \left.-2 e_{105} v_{\mu \nu}^{(s)} \operatorname{Tr}\left(\chi_{+}\right)-\frac{1}{2} e_{106} f_{\mu \nu}^{+} \operatorname{Tr}\left(\chi_{+}\right)\right] \sigma^{\mu \nu} \Psi+\cdots
\end{aligned}
$$

The values for the low-energy constants (LECs) have to be determined from empirical input. In general, their numerical values depend on the renormalization scheme in question. As we will see later, some of the constants can be fitted to the anomalous magnetic moments and to the charge and magnetic radii of the nucleon.

\section{ELECTROMAGNETIC FORM FACTORS OF THE NUCLEON}

\section{A. Definition of the electromagnetic form factors}

In terms of QCD degrees of freedom the interaction with an external electromagnetic field $\mathcal{A}_{\mu}$ is given by

$$
\mathcal{L}_{\text {e.m. }}=-e J^{\mu} \mathcal{A}_{\mu}
$$

where the electromagnetic current operator reads

$$
J^{\mu}(x)=\frac{2}{3} \bar{u}(x) \gamma^{\mu} u(x)-\frac{1}{3} \bar{d}(x) \gamma^{\mu} d(x)+\cdots=\bar{q}(x)\left(\frac{1}{6}+\frac{\tau_{3}}{2}\right) \gamma^{\mu} q(x)+\cdots .
$$

The ellipsis denotes the contribution due to the heavier quarks which we do not consider here. The electromagnetic form factors are defined via the matrix element

$$
\left\langle N\left(p_{f}\right)\left|J^{\mu}(0)\right| N\left(p_{i}\right)\right\rangle=\bar{u}\left(p_{f}\right)\left[\gamma^{\mu} F_{1}^{N}\left(Q^{2}\right)+\frac{i \sigma^{\mu \nu} q_{\nu}}{2 m_{N}} F_{2}^{N}\left(Q^{2}\right)\right] u\left(p_{i}\right), \quad N=p, n,
$$

where $q=p_{f}-p_{i}$ is the momentum transfer and $Q^{2} \equiv-q^{2}=-t \geq 0 .^{3}$ The functions $F_{1}^{N}\left(Q^{2}\right)$ and $F_{2}^{N}\left(Q^{2}\right)$ are the Dirac and Pauli form factors of the nucleon, respectively. At $Q^{2}=0$, these form factors are given by the electric charges and the anomalous magnetic moments in units of the charge and the nuclear magneton, respectively:

$$
F_{1}^{p}(0)=1, \quad F_{1}^{n}(0)=0, \quad F_{2}^{p}(0)=\kappa_{p}=1.793, \quad F_{2}^{n}(0)=\kappa_{n}=-1.913 .
$$

In that sense, Eq. (6) provides a "natural" extension of the electromagnetic vertex of a "point particle" with an anomalous magnetic moment. ${ }^{4}$ In the actual calculation, it is more

\footnotetext{
${ }^{2}$ We took the Lagrangians of Ref. [9] and made the replacements $\tilde{F}_{\mu \nu}^{+} \rightarrow f_{\mu \nu}^{+}$and $\operatorname{Tr}\left(F_{\mu \nu}^{+}\right) \rightarrow 4 v_{\mu \nu}^{(s)}$.

${ }^{3}$ Since we discuss the form factors in the space-like region, here we adopt the convention of taking $Q^{2}=-t$ as the argument of the form factors as is common practice in the context of electron scattering.

${ }^{4}$ For a discussion of the implications of gauge invariance in its strong form and alternative forms of the vertex, see, e.g., Ref. [36].
} 
convenient to work in the isospin basis

$$
F_{i}^{(s)}=F_{i}^{p}+F_{i}^{n}, \quad F_{i}^{(v)}=F_{i}^{p}-F_{i}^{n}, \quad i=1,2,
$$

so that the electromagnetic form factors are obtained as follows,

$$
F_{i}^{N}=\frac{1}{2} F_{i}^{(s)}+\frac{\tau_{3}}{2} F_{i}^{(v)}, \quad i=1,2 .
$$

Experimental data are usually analyzed in terms of the electric and magnetic Sachs form factors [18, 19], $G_{E}^{N}\left(Q^{2}\right)$ and $G_{M}^{N}\left(Q^{2}\right)$, defined by

$$
\begin{aligned}
& G_{E}^{N}\left(Q^{2}\right)=F_{1}^{N}\left(Q^{2}\right)-\frac{Q^{2}}{4 m_{N}^{2}} F_{2}^{N}\left(Q^{2}\right), \\
& G_{M}^{N}\left(Q^{2}\right)=F_{1}^{N}\left(Q^{2}\right)+F_{2}^{N}\left(Q^{2}\right) .
\end{aligned}
$$

The Fourier transforms of the Sachs form factors in the Breit frame can be related to the distribution of charge and magnetization inside the nucleon. For a recent discussion of the limits of such an interpretation, see Ref. [20].

\section{B. Calculation of the Dirac and Pauli form factors}

The graphs contributing to the electromagnetic current matrix element of Eq. (6) up to and including $\mathcal{O}\left(q^{4}\right)$ are shown in Fig. 1 (we do not display external leg corrections). ${ }^{5}$ In particular, the loop graphs labeled by the numbers (5) to (9) in Fig. 1 contribute at $\mathcal{O}\left(q^{3}\right)$ while the graphs $(10)$ to $(12)$ contribute at $\mathcal{O}\left(q^{4}\right)$. The individual unrenormalized contributions to the form factors $F_{1}$ and $F_{2}$ can be found in App. B. To renormalize the form factor diagrams we first apply the subtraction scheme used by Gasser and Leutwyler 2, 4] which we denote by modified minimal subtraction scheme of $\chi \mathrm{PT}(\widetilde{\mathrm{MS}})$. We then perform the additional finite subtractions according to our EOMS scheme (see Ref. [17] for details). For this we determine the chiral order of a given diagram by applying the standard power counting and then subtract those terms which violate the power counting. Such finite subtractions are achieved by expanding the quantities of the $\overline{\mathrm{MS}}$ scheme in terms of the parameters of our EOMS scheme. This expansion produces the counterterm contributions which are responsible for the required additional subtractions. In view of Eq. (6) , an $\mathcal{O}\left(q^{4}\right)$ calculation of the electromagnetic form factors yields $F_{1}$ to $\mathcal{O}\left(q^{3}\right)$ and $F_{2}$ to $\mathcal{O}\left(q^{2}\right)$, since both the polarization vector $\epsilon^{\mu}$ and the four-momentum $q^{\mu}$ of the virtual photon count as $\mathcal{O}(q)$. Diagrams potentially violating the power counting are loop diagrams with internal nucleon lines. In the present case, we find that the diagrams (5), (8), and (10) of Fig. 11 need subtractions beyond $\widetilde{\mathrm{MS}}$ whereas this is not necessary for the diagrams (7). The subtraction term for the Dirac form factor reads

$$
\Delta F_{1}^{10}=\frac{{\stackrel{\circ}{g_{A}}}^{2} m}{64 \pi^{2} F^{2}}\left(3 c_{7}-2 c_{6} \tau_{3}\right) t
$$

\footnotetext{
${ }^{5}$ In order to take care of the mass shift due to $\mathcal{L}_{\pi N}^{(2)}$, we use $m_{2}=m-4 c_{1} M^{2}$ in the fermion propagators 12. At the end of the calculation we then replace $m_{2}$ by the physical nucleon mass $m_{N}$, because the difference shows up only in higher orders.
} 
and similarly for the Pauli form factor ${ }^{6}$

$$
\begin{aligned}
& \Delta F_{2}^{5}=-\frac{{\stackrel{\circ}{g_{A}}}^{2} m_{N}\left(m-4 c_{1} M^{2}\right)}{32 \pi^{2} F^{2}}\left(3-\tau_{3}\right), \\
& \Delta F_{2}^{8}=\frac{{\stackrel{\circ}{g_{A}}}^{2} m_{N}\left(m-4 c_{1} M^{2}\right)}{8 \pi^{2} F^{2}} \tau_{3}, \\
& \Delta F_{2}^{10}=-\frac{\stackrel{\circ}{A}^{2} m_{N}\left(m^{2}-8 c_{1} M^{2} m\right)}{16 \pi^{2} F^{2}}\left(3 c_{7}-2 c_{6} \tau_{3}\right) \text {. }
\end{aligned}
$$

For completeness, we determine the expansion of the couplings of the $\widetilde{\mathrm{MS}}$ scheme $c_{6}^{r}, c_{7}^{r}, d_{6}^{r}$, $d_{7}^{r}, e_{54}^{r}$, and $e_{74}^{r}$ in terms of our renormalized parameters, ${ }^{7}$

$$
\begin{aligned}
& c_{6}^{r}=c_{6}-\frac{{\stackrel{\circ}{g_{A}}}^{2} m}{64 \pi^{2} F^{2}}\left(5+4 m c_{6}\right) \text {, } \\
& c_{7}^{r}=c_{7}+\frac{3{\stackrel{\circ}{g_{A}}}^{2} m}{32 \pi^{2} F^{2}}\left(1+2 m c_{7}\right) \text {, } \\
& d_{6}^{r}=d_{6}-\frac{\stackrel{\circ g_{A}^{2}}{ }{ }^{2} m}{32 \pi^{2} F^{2}} c_{6}, \\
& d_{7}^{r}=d_{7}+\frac{3{\stackrel{\circ}{g_{A}}}^{2} m}{128 \pi^{2} F^{2}} c_{7}, \\
& e_{54}^{r}=e_{54}-\frac{3 \stackrel{\circ}{g_{A}^{2}}}{256 \pi^{2} F^{2}} c_{7} \text {, } \\
& e_{74}^{r}=e_{74}+\frac{\stackrel{\circ}{g}^{2}}{64 \pi^{2} F^{2}} c_{6}, \\
& e_{105}^{r}=e_{105}+\frac{{\stackrel{\circ}{g_{A}}}^{2} c_{1}}{128 \pi^{2} F^{2}}\left(3+12 m c_{7}\right) \text {, } \\
& e_{106}^{r}=e_{106}-\frac{{\stackrel{\circ}{g_{A}}}^{2} c_{1}}{64 \pi^{2} F^{2}}\left(5+8 m c_{6}\right) \text {, }
\end{aligned}
$$

producing all the required counterterm contributions.

In order to obtain the final expression one has to sum up all contributions and to multiply the result with the wave function renormalization constant $Z,{ }^{8}$ given by [17]

$$
Z=1-\frac{9{\stackrel{\circ}{g_{A}}}^{2} M^{2}}{32 \pi^{2} F^{2}}\left[\frac{2}{3}+\ln \left(\frac{M}{m}\right)\right]+\frac{9{\stackrel{\circ}{g_{A}}}^{2} M^{3}}{64 \pi F^{2} m}+O\left(M^{4}\right) \text {. }
$$

Next, we need to fix the parameters in order to get a graphical representation of the form factors. The values of the renormalized LECs $c_{2}$ and $c_{4}$ are taken from a (tree-level) fit [37]

\footnotetext{
${ }^{6}$ One factor of $m_{N}$ is due to the fact that the parameterization of Eq. (6) contains an explicit factor $1 / m_{N}$ multiplying the Pauli form factor.

${ }^{7}$ For notational convenience, we omit a subscript $\mathrm{R}$ for the EOMS-renormalized parameters, but one should not confuse them with the bare parameters of the bare Lagrangian.

${ }^{8}$ For $F_{2}$, one needs $Z$ only up to order $\mathcal{O}\left(q^{2}\right)$ due to the factor $q_{\nu}$ in Eq. (6).
} 
to the $\pi N$ scattering threshold parameters of Ref. [38]: $c_{2}=2.5 m_{N}^{-1}$ and $c_{4}=2.3 m_{N}^{-1}$. Since the $c_{i}$ only enter loop expressions at $\mathcal{O}\left(q^{4}\right)$ a determination at $\mathcal{O}\left(q^{2}\right)$ is consistent with the present accuracy.

The chiral expansion of the anomalous isoscalar and isovector magnetic moments can be written as

$$
\kappa^{(s / v)}=\kappa_{0}^{(s / v)}+\kappa_{1}^{(s / v)} M+\kappa_{2}^{(s / v)} M^{2} \ln \left(\frac{M}{m}\right)+\kappa_{3}^{(s / v)} M^{2}+O\left(M^{3}\right)
$$

where the coefficients, in terms of the EOMS-renormalized parameters, are given by

$$
\begin{aligned}
& \kappa_{0}^{(s)}=2 c_{7} m \\
& \kappa_{1}^{(s)}=0, \\
& \kappa_{2}^{(s)}=-\frac{3\left(1+2 c_{7} m\right){\stackrel{\circ}{g_{A}}}^{2}}{8 \pi^{2} F^{2}}, \\
& \kappa_{3}^{(s)}=-\frac{3\left(2+3 c_{7} m\right){\stackrel{\circ}{g_{A}}}^{2}}{16 \pi^{2} F^{2}}-8 c_{1} c_{7}-32 e_{105} m, \\
& \kappa_{0}^{(v)}=4 c_{6} m, \\
& \kappa_{1}^{(v)}=-\frac{\circ_{A}^{2} m}{4 \pi F^{2}}, \\
& \kappa_{2}^{(v)}=\frac{4\left(c_{4}-c_{6}\right) m-\circ_{A}^{2}\left(7+8 c_{6} m\right)}{8 \pi^{2} F^{2}}, \\
& \kappa_{3}^{(v)}=-\frac{\stackrel{\circ}{A}^{2}\left(1+5 c_{6} m\right)}{8 \pi^{2} F^{2}}-16 c_{1} c_{6}-16 e_{106} m .
\end{aligned}
$$

Comparing with Ref. 33] we see that the lowest-order terms as well as the non-analytic terms $\sim M \sim \hat{m}^{1 / 2}$ and $\sim \ln (M)$ coincide, while the analytic $\kappa_{3}^{(s / v)}$ terms differ, because we use a different renormalization scheme.

Since chiral perturbation theory does not predict the anomalous magnetic moments, we take the empirical values as obtained from Eq. (7) to fix the Pauli form factors at $Q^{2}=0$. The constants $d_{6}, d_{7}, e_{54}$, and $e_{74}$ were obtained from the charge and magnetic radii of the nucleon, which are defined via the Sachs form factors [see Eqs. (9) and (10)]:

$$
\begin{array}{lll}
\left\langle\left(r_{E}^{p}\right)^{2}\right\rangle=-\left.\frac{6}{G_{E}^{p}(0)} \frac{d G_{E}^{p}\left(Q^{2}\right)}{d Q^{2}}\right|_{Q^{2}=0}, & \left\langle\left(r_{M}^{p}\right)^{2}\right\rangle=-\left.\frac{6}{G_{M}^{p}(0)} \frac{d G_{M}^{p}\left(Q^{2}\right)}{d Q^{2}}\right|_{Q^{2}=0}, \\
\left\langle\left(r_{E}^{n}\right)^{2}\right\rangle=-\left.6 \frac{d G_{E}^{n}\left(Q^{2}\right)}{d Q^{2}}\right|_{Q^{2}=0}, & \left\langle\left(r_{M}^{n}\right)^{2}\right\rangle=-\left.\frac{6}{G_{M}^{n}(0)} \frac{d G_{M}^{n}\left(Q^{2}\right)}{d Q^{2}}\right|_{Q^{2}=0} .
\end{array}
$$

For the numerical analysis, we make use of the charge and magnetic radii as obtained in the dispersion-theoretical analysis of Ref. [28], $r_{E}^{p}=0.847 \mathrm{fm}, r_{E}^{n}=-0.113 \mathrm{fm}, r_{M}^{p}=0.836 \mathrm{fm}$ and $r_{M}^{n}=0.889 \mathrm{fm}$. Within the accuracy of our calculation the constants $c_{6}, c_{7}, e_{105}$, and $e_{106}$ appear in the combinations

$$
\tilde{c}_{6}=c_{6}-4 M^{2} e_{106}, \quad \tilde{c}_{7}=c_{7}-16 M^{2} e_{105} .
$$

Therefore for the numerical analysis we only need the values for the combinations $\tilde{c}_{6,7}$. The values for the LECs are summarized in Table \. From Table \we see that there is quite a big 
difference in the value for the constant $d_{6}$ as obtained in the infrared regularization and our renormalization scheme, respectively. The constants $d_{6}$ and $d_{7}$ yield the leading order of the electromagnetic radii and thus determine, together with those loop graphs which depend on $Q^{2}$, the slope of the graphs at the origin.

\begin{tabular}{|c||c|c|c|c|c|c|c|c|}
\hline & $c_{2}$ & $c_{4}$ & $\tilde{c}_{6}$ & $\tilde{c}_{7}$ & $d_{6}$ & $d_{7}$ & $e_{54}$ & $e_{74}$ \\
\hline \hline EOMS & 2.66 & 2.45 & 1.26 & -0.13 & -0.69 & -0.50 & 0.19 & 1.59 \\
IR & 2.66 & 2.45 & 1.26 & -0.18 & 0.54 & -0.73 & 0.25 & 1.93 \\
\hline
\end{tabular}

TABLE I: Values for the relevant low-energy constants in the extended on-mass-shell renormalization scheme (EOMS) and in the infrared regularization scheme (IR) within an $\mathcal{O}\left(q^{4}\right)$ calculation of relativistic chiral perturbation theory. The LECs $c_{i}$ are given in units of $\mathrm{GeV}^{-1}$, the $d_{i}$ in units of $\mathrm{GeV}^{-2}$, and the $e_{i}$ in units of $\mathrm{GeV}^{-3}$.

The results for the Dirac and Pauli form factors are shown in Fig. 2 for momentum transfers $0 \leq Q^{2} \leq 0.4 \mathrm{GeV}^{2}$. For comparison the results of the infrared regularization 33. are also shown. We see from Fig. 2 that for the Dirac form factor of the proton and for both Pauli form factors the two methods generate very similar results. However, this is not the case for the Dirac form factor of the neutron which is very small in the entire small $Q^{2}$ region. We conclude that $F_{1}^{n}$ significantly depends on higher-order contributions.

As an example, let us have a closer look at the Dirac form factor of the proton, $F_{1}^{p}$, which, for both methods, shows an almost linear behavior in $Q^{2}$, i.e., has very little curvature. In order to show the influence of the loop contributions, in Fig. 3] we compare the full results for $F_{1}^{p}$ in both relativistic renormalization schemes with the contact-graph contributions only. We see that the loop contributions cannot be neglected, i.e., the contribution of the pion cloud plays an important role. Moreover, in the infrared regularization the main contribution to the slope is due to loop contributions, while in our renormalization scheme the loop contributions are smaller. The situation here is different from the electromagnetic form factor of the pion whose linear behavior is predominantly due to a contact graph [2].

\section{Results for the Sachs form factors}

We now turn to a discussion of the Sachs form factors in the EOMS renormalization scheme and compare them with experimental data as well as the results of the infrared regularization and of $\mathrm{HB} \chi \mathrm{PT}$ at $\mathcal{O}\left(q^{3}\right)[6,31] .{ }^{9}$ Recall that a full $\mathcal{O}\left(q^{4}\right)$ calculation yields $G_{E}$ and $G_{M}$ up to $\mathcal{O}\left(q^{3}\right)$ and $\mathcal{O}\left(q^{2}\right)$, respectively. ${ }^{10}$

In order to discuss the Sachs form factors resulting from an $\mathcal{O}\left(q^{3}\right)$ calculation, we need to recalculate the adapted values for the LECs (see Table II) since the values of Table (1) were obtained from an $\mathcal{O}\left(q^{4}\right)$ calculation. Note that the changes in the LECs turn out to be reasonably small. Figure 4 shows our results for the Sachs form factors at $\mathcal{O}\left(q^{3}\right)$ together with experimental data as well as the infrared regularization and $\mathrm{HB} \chi \mathrm{PT}$ results

\footnotetext{
${ }^{9}$ The effects due to the $\Delta$ resonance, as calculated in the small-scale expansion, can be found in Ref. 32 .

${ }^{10}$ As discussed in Ref. 33], when combining $F_{1}$ and $F_{2}$ to give the Sachs form factors $G_{E}$ and $G_{M}$ [see Eqs. (9) and [10] the chiral orders are mixed.
} 


\begin{tabular}{|c||c|c|c|c|}
\hline & $c_{6}$ & $c_{7}$ & $d_{6}$ & $d_{7}$ \\
\hline \hline EOMS & 1.34 & -0.15 & -0.70 & -0.49 \\
IR & 1.36 & -0.23 & 0.50 & -0.73 \\
\hline
\end{tabular}

TABLE II: Values for the relevant low-energy constants in the extended on-mass-shell renormalization scheme (EOMS) and in the infrared regularization scheme (IR) within an $\mathcal{O}\left(q^{3}\right)$ calculation of relativistic chiral perturbation theory. The LECs $c_{i}$ are given in units of $\mathrm{GeV}^{-1}$ and the $d_{i}$ in units of $\mathrm{GeV}^{-2}$.

at $\mathcal{O}\left(q^{3}\right)$. For both Sachs form factors of the proton and the magnetic Sachs form factor of the neutron the two relativistic calculations are almost identical while the $\mathrm{HB} \chi \mathrm{PT}$ results clearly deviate from the relativistic calculations. This difference may be interpreted as being due to relativistic corrections which, in $\mathrm{HB} \chi \mathrm{PT}$, would have to show up in higher orders. It is somewhat surprising that in all three cases the $\mathrm{HB} \chi \mathrm{PT}$ results are closer to the experimental data. The slopes of the electric form factors are essentially determined by fitting the mean square electric radii. On the other hand, at $\mathcal{O}\left(q^{3}\right)$ the magnetic radii do not contain any free parameters. This explains the difference in the behavior of the slopes of the magnetic form factors between the relativistic calculation on the one hand and the $\mathrm{HB} \chi \mathrm{PT}$ calculation on the other hand, originating from the fact that the relativistic calculations at $\mathcal{O}\left(q^{3}\right)$ for the magnetic radii also contain higher-order terms in $M$, whereas $\mathrm{HB} \chi \mathrm{PT}$ only involves the leading-order term. Finally, the electric form factor of the neutron is very small and all three theoretical curves disagree. Here, the relativistic $\mathcal{O}\left(q^{3}\right)$ calculations are in better agreement with the experimental data.

Figure 5] shows the results of the two relativistic calculations for the Sachs form factors at $\mathcal{O}\left(q^{4}\right)$. The description of $G_{E}^{p}, G_{M}^{p}$, and $G_{M}^{n}$ is only marginally better than that of the $\mathcal{O}\left(q^{3}\right)$ calculation. For the very-small $Q^{2}$ region this is due to additional free parameters which have been adjusted to the magnetic radii. Clearly, the $\mathcal{O}\left(q^{4}\right)$ results do not generate sufficient curvature which is emphasized in Figs. 6, 7] and 8, showing the form factors and data divided by the dipole form factor $G_{D}$ and normalized to one at the origin. Finally, the situation for $G_{E}^{n}$ seems to be even worse, where we find better agreement with the experimental data for the $\mathcal{O}\left(q^{3}\right)$ results. We conclude that the perturbation series converges, at best, slowly and that higher-order contributions must play an important role.

\section{SUMMARY}

We have calculated the electromagnetic form factors of the nucleon at $\mathcal{O}\left(q^{4}\right)$ (one-loop order) in relativistic chiral perturbation theory using the extended on-mass-shell renormalization scheme of Ref. [17]. The relevant low-energy coupling constants have been fitted to the anomalous magnetic moments and to the charge and magnetic mean square radii. As a general trend the results for both the proton form factors $G_{E}^{p}$ and $G_{M}^{p}$ and the magnetic neutron form factor $G_{M}^{n}$ do not show sufficient curvature to generate agreement with the experimental data beyond small values of $Q^{2} \sim 0.1 \mathrm{GeV}^{2}$. Our results are very similar to those of the infrared regularization [33] and the small differences between the two methods are related to the way how the regular higher-order terms of loop integrals are treated. In the case of the electric neutron form factor $G_{E}^{n}$, in both renormalization schemes the de- 
scription does not seem to improve when going from $\mathcal{O}\left(q^{3}\right)$ to $\mathcal{O}\left(q^{4}\right)$. We conclude that a relativistic treatment at the one-loop level using nucleon and pion degrees of freedom only is not sufficient to describe the form factors for $Q^{2} \geq 0.1 \mathrm{GeV}^{2}$ and that higher-order contributions must play an important role. Such higher-order contributions either have to be studied in the framework of a two-loop calculation or by explicitly including other dynamical degrees of freedom such as vector mesons [33]. We stress that the EOMS renormalization scheme allows for also implementing a consistent power counting in relativistic baryon chiral perturbation theory when vector (and axial-vector) mesons are explicitly included [56].

\section{Acknowledgments}

The work of T.F. and S.S. was supported by the Deutsche Forschungsgemeinschaft (SFB 443). J.G. acknowledges the support of the Alexander von Humboldt Foundation.

\section{APPENDIX A: LOOP INTEGRALS}

\section{Definition of the loop integrals}

In the appendix we use the following notation

$$
P^{\mu}=p_{i}^{\mu}+p_{f}^{\mu}, \quad q^{\mu}=p_{f}^{\mu}-p_{i}^{\mu}, \quad t=q^{2} .
$$

The loop integrals with one and two internal lines are defined as follows:

$$
\begin{gathered}
I_{\pi}=i \int \frac{d^{n} k}{(2 \pi)^{n}} \frac{1}{k^{2}-M^{2}+i \epsilon}, \\
I_{N}=i \int \frac{d^{n} k}{(2 \pi)^{n}} \frac{1}{k^{2}-m^{2}+i \epsilon}, \\
I_{\pi \pi}(t)=i \int \frac{d^{n} k}{(2 \pi)^{n}} \frac{1}{\left[k^{2}-M^{2}+i \epsilon\right]\left[(k+q)^{2}-M^{2}+i \epsilon\right]}, \\
q^{\mu} I_{\pi \pi}^{(q)}(t)=i \int \frac{d^{n} k}{(2 \pi)^{n}} \frac{k^{\mu}}{\left[g^{\mu \nu}-M^{2}+i \epsilon\right]\left[(k+q)^{2}-M^{2}+i \epsilon\right]}, \\
I_{\pi \pi}^{(00)}(t)+q^{\mu} q^{\nu} I_{\pi \pi}^{(q q)}(t)=i \int \frac{d^{n} k}{(2 \pi)^{n}} \frac{k^{\mu} k^{\nu}}{\left[k^{2}-M^{2}+i \epsilon\right]\left[(k+q)^{2}-M^{2}+i \epsilon\right]}, \\
I_{\pi N}(t)=i \int \frac{d^{n} k}{(2 \pi)^{n}} \frac{1}{\left[k^{2}-m^{2}+i \epsilon\right]\left[(k+q)^{2}-m^{2}+i \epsilon\right]}, \\
p^{\mu} I_{\pi N}^{(p)}\left(p^{2}\right)=i \int \frac{d^{n} k}{(2 \pi)^{n}} \frac{1}{\left[k^{2}-M^{2}+i \epsilon\right]\left[(k-p)^{2}-m^{2}+i \epsilon\right]}, \\
k^{\mu}
\end{gathered}
$$

In the following loop integrals involving three internal lines we always assume on-shell kinematics, $p_{i}^{2}=p_{f}^{2}=m_{N}^{2}$,

$$
I_{\pi \pi N}(t)=i \int \frac{d^{n} k}{(2 \pi)^{n}} \frac{1}{\left[k^{2}-M^{2}+i \epsilon\right]\left[(k+q)^{2}-M^{2}+i \epsilon\right]\left[\left(k-p_{i}\right)^{2}-m^{2}+i \epsilon\right]},
$$




$$
\begin{gathered}
P^{\mu} I_{\pi \pi N}^{(P)}(t)-\frac{1}{2} q^{\mu} I_{\pi \pi N}(t)=i \int \frac{d^{n} k}{(2 \pi)^{n}} \frac{k^{\mu}}{\left[k^{2}-M^{2}+i \epsilon\right]\left[(k+q)^{2}-M^{2}+i \epsilon\right]\left[\left(k-p_{i}\right)^{2}-m^{2}+i \epsilon\right]}, \\
g^{\mu \nu} I_{\pi \pi N}^{(00)}(t)+P^{\mu} P^{\nu} I_{\pi \pi N}^{(P P)}(t)+q^{\mu} q^{\nu} I_{\pi \pi N}^{(q q)}(t)-\frac{q^{\mu} P^{\nu}+P^{\mu} q^{\nu}}{2} I_{\pi \pi N}^{(P)}(t) \\
=i \int \frac{d^{n} k}{(2 \pi)^{n}} \frac{k^{\mu} k^{\nu}}{\left[k^{2}-M^{2}+i \epsilon\right]\left[(k+q)^{2}-M^{2}+i \epsilon\right]\left[\left(k-p_{i}\right)^{2}-m^{2}+i \epsilon\right]}, \\
I_{\pi N N}(t)=i \int \frac{d^{n} k}{(2 \pi)^{n}} \frac{1}{\left[k^{2}-M^{2}+i \epsilon\right]\left[\left(k-p_{i}\right)^{2}-m^{2}+i \epsilon\right]\left[\left(k-p_{f}\right)^{2}-m^{2}+i \epsilon\right]}, \\
P^{\mu} I_{\pi N N}^{(P)}(t)=i \int \frac{d^{n} k}{(2 \pi)^{n}} \frac{k^{\mu}}{\left[k^{2}-M^{2}+i \epsilon\right]\left[\left(k-p_{i}\right)^{2}-m^{2}+i \epsilon\right]\left[\left(k-p_{f}\right)^{2}-m^{2}+i \epsilon\right]}, \\
g^{\mu \nu} I_{\pi N N}^{(00)}(t)+P^{\mu} P^{\nu} I_{\pi N N}^{(P P)}(t)+q^{\mu} q^{\nu} I_{\pi N N}^{(q q)}(t) \\
=i \int \frac{d^{n} k}{(2 \pi)^{n}} \frac{k^{\mu} k^{\nu}}{\left[k^{2}-M^{2}+i \epsilon\right]\left[\left(k-p_{i}\right)^{2}-m^{2}+i \epsilon\right]\left[\left(k-p_{f}\right)^{2}-m^{2}+i \epsilon\right]} .
\end{gathered}
$$

\section{Scalar loop integrals}

Defining

$$
\bar{\lambda}=\frac{m^{n-4}}{16 \pi^{2}}\left\{\frac{1}{n-4}-\frac{1}{2}\left[\ln (4 \pi)+\Gamma^{\prime}(1)+1\right]\right\}
$$

and

$$
\Omega=\frac{p^{2}-m^{2}-M^{2}}{2 m M},
$$

the scalar loop integrals are given by 17

$$
\begin{gathered}
I_{\pi}=2 M^{2} \bar{\lambda}+\frac{M^{2}}{8 \pi^{2}} \ln \left(\frac{M}{m}\right) \\
I_{N}=2 m^{2} \bar{\lambda} \\
I_{\pi \pi}(t)=2 \bar{\lambda}+\frac{1}{16 \pi^{2}}\left[1+2 \ln \left(\frac{M}{m}\right)+J^{(0)}\left(\frac{t}{M^{2}}\right)\right] \\
I_{N N}(t)=2 \bar{\lambda}+\frac{1}{16 \pi^{2}}\left[1+J^{(0)}\left(\frac{t}{m^{2}}\right)\right] \\
I_{\pi N}\left(p^{2}\right)=2 \bar{\lambda}+\frac{1}{16 \pi^{2}}\left[-1+\frac{p^{2}-m^{2}+M^{2}}{p^{2}} \ln \left(\frac{M}{m}\right)+\frac{2 m M}{p^{2}} F(\Omega)\right],
\end{gathered}
$$

where

$$
\begin{aligned}
J^{(0)}(x) & =\int_{0}^{1} d z \ln \left[1+x\left(z^{2}-z\right)-i \epsilon\right] \\
& = \begin{cases}-2-\sigma \ln \left(\frac{\sigma-1}{\sigma+1}\right), & x<0, \\
-2+2 \sqrt{\frac{4}{x}-1} \operatorname{arccot}\left(\sqrt{\frac{4}{x}-1}\right), & 0 \leq x<4, \\
-2-\sigma \ln \left(\frac{1-\sigma}{1+\sigma}\right)-i \pi \sigma, & 4<x,\end{cases}
\end{aligned}
$$


with

$$
\sigma(x)=\sqrt{1-\frac{4}{x}}, \quad x \notin[0,4],
$$

and

$$
F(\Omega)= \begin{cases}\sqrt{\Omega^{2}-1} \ln \left(-\Omega-\sqrt{\Omega^{2}-1}\right), & \Omega \leq-1 \\ \sqrt{1-\Omega^{2}} \arccos (-\Omega), & -1 \leq \Omega \leq 1 \\ \sqrt{\Omega^{2}-1} \ln \left(\Omega+\sqrt{\Omega^{2}-1}\right)-i \pi \sqrt{\Omega^{2}-1}, & 1 \leq \Omega\end{cases}
$$

In our numerical analysis, we made use of a parameterization in terms of a Feynman parameter integral to analyze the loop integrals with three internal lines. Such integrals may also be determined using a dispersion relation (see App. C of Ref. [17]).

\section{Reduction of the tensorial loop integrals}

The reduction of the tensorial loop integrals to the corresponding scalar ones can be performed in the standard way [57]. We obtain the following results:

$$
\begin{aligned}
& I_{\pi \pi}^{(q)}(t)=-\frac{1}{2} I_{\pi \pi}(t) \\
& I_{\pi \pi}^{(q q)}(t)=\frac{1}{(n-1) t}\left[\frac{n-2}{2} I_{\pi}+\left(\frac{n}{4} t-M^{2}\right) I_{\pi \pi}(t)\right] \\
& =\frac{1}{t}\left[\frac{1}{3} I_{\pi}+\frac{1}{3}\left(t-M^{2}\right) I_{\pi \pi}(t)+\frac{1}{144 \pi^{2}}\left(3 M^{2}-\frac{t}{2}\right)\right], \\
& I_{\pi \pi}^{(00)}(t)=\frac{1}{(n-1) t}\left[\frac{1}{2} I_{\pi}+\frac{1}{4}\left(4 M^{2}-t\right) I_{\pi \pi}(t)\right] \\
& =\frac{1}{t}\left[\frac{1}{6} I_{\pi}(t)+\frac{1}{12}\left(4 M^{2}-t\right) I_{\pi \pi}(t)-\frac{1}{8 \pi^{2}}\left(\frac{M^{2}}{6}-\frac{t}{36}\right)\right], \\
& I_{\pi N}^{(p)}\left(p^{2}\right)=\frac{1}{2 p^{2}}\left[I_{N}-I_{\pi}+\left(p^{2}-m^{2}+M^{2}\right) I_{\pi N}\left(p^{2}\right)\right], \\
& I_{\pi \pi N}^{(P)}(t)=\frac{1}{2\left(4 m_{N}^{2}-t\right)}\left[\left(2 M^{2}-t\right) I_{\pi \pi N}(t)+2 I_{\pi N}\left(m_{N}^{2}\right)-2 I_{\pi \pi}(t)\right], \\
& I_{\pi \pi N}^{(00)}(t)=\frac{1}{4(n-2)}\left[2 I_{\pi N}\left(m_{N}^{2}\right)+\left(4 M^{2}-t\right) I_{\pi \pi N}(t)-2\left(2 M^{2}-t\right) I_{\pi \pi N}^{(P)}(t)\right] \\
& =\frac{1}{8}\left[2 I_{\pi N}\left(m_{N}^{2}\right)+\left(4 M^{2}-t\right) I_{\pi \pi N}(t)-2\left(2 M^{2}-t\right) I_{\pi \pi N}^{(P)}(t)-\frac{1}{8 \pi^{2}}\right], \\
& I_{\pi \pi N}^{(P P)}(t)=\frac{1}{4(n-2)\left(4 m_{N}^{2}-t\right)}\left[-2 I_{\pi N}\left(m_{N}^{2}\right)+2(n-2) I_{\pi N}^{(p)}\left(m_{N}^{2}\right)-\left(4 M^{2}-t\right) I_{\pi \pi N}(t)\right.
\end{aligned}
$$




$$
\begin{aligned}
& \left.+2(n-1)\left(2 M^{2}-t\right) I_{\pi \pi N}^{(P)}(t)\right] \\
& =\frac{1}{8\left(4 m_{N}^{2}-t\right)}\left[-2 I_{\pi N}\left(m_{N}^{2}\right)+4 I_{\pi N}^{(p)}\left(m_{N}^{2}\right)-\left(4 M^{2}-t\right) I_{\pi \pi N}(t)\right. \\
& \left.+6\left(2 M^{2}-t\right) I_{\pi \pi N}^{(P)}(t)+\frac{1}{8 \pi^{2}}\right] \\
& I_{\pi \pi N}^{(q q)}(t)=\frac{1}{4(n-2) t}\left\{2(n-3) I_{\pi N}\left(m_{N}^{2}\right)-2(n-2) I_{\pi N}^{(p)}\left(m_{N}^{2}\right)\right. \\
& \left.-\left[4 M^{2}-(n-1) t\right] I_{\pi \pi N}(t)+2\left(2 M^{2}-t\right) I_{\pi \pi N}^{(P)}(t)\right\} \\
& =\frac{1}{8 t}\left[2 I_{\pi N}\left(m_{N}^{2}\right)-4 I_{\pi N}^{(p)}\left(m_{N}^{2}\right)-\left(4 M^{2}-3 t\right) I_{\pi \pi N}(t)+2\left(2 M^{2}-t\right) I_{\pi \pi N}^{(P)}(t)+\frac{1}{8 \pi^{2}}\right], \\
& I_{\pi N N}^{(P)}(t)=\frac{1}{4 m^{2}-t}\left[M^{2} I_{\pi N N}(t)-I_{\pi N}\left(m_{N}^{2}\right)+I_{N N}(t)\right] \\
& I_{\pi N N}^{(00)}(t)=\frac{1}{n-2}\left\{\left[I_{\pi N N}(t)-I_{\pi N N}^{(P)}(t)\right] M^{2}+\frac{1}{2} I_{N N}(t)\right\} \\
& =\frac{1}{2}\left\{\left[I_{\pi N N}(t)-I_{\pi N N}^{(P)}(t)\right] M^{2}+\frac{1}{2} I_{N N}(t)-\frac{1}{32 \pi^{2}}\right\}, \\
& I_{\pi N N}^{(P P)}(t)=\frac{1}{(n-2)\left(4 m_{N}^{2}-t\right)}\left\{\left[(n-1) I_{\pi N N}^{(P)}(t)-I_{\pi N N}(t)\right] M^{2}-\frac{n-2}{2} I_{\pi N}^{(p)}\left(m_{N}^{2}\right)+\frac{n-3}{2} I_{N N}(t)\right\} \\
& =\frac{1}{2\left(4 m_{N}^{2}-t\right)}\left\{\left[3 I_{\pi N N}^{(P)}(t)-I_{\pi N N}(t)\right] M^{2}-I_{\pi N}^{(p)}\left(m_{N}^{2}\right)+\frac{1}{2} I_{N N}(t)+\frac{1}{32 \pi^{2}}\right\}, \\
& I_{\pi N N}^{(q q)}(t)=\frac{1}{(n-2) t}\left\{\left[I_{\pi N N}^{(P)}(t)-I_{\pi N N}(t)\right] M^{2}+\frac{n-2}{2} I_{\pi N}^{(p)}\left(m_{N}^{2}\right)-\frac{1}{2} I_{N N}(t)\right\} \\
& =\frac{1}{2 t}\left\{\left[I_{\pi N N}^{(P)}(t)-I_{\pi N N}(t)\right] M^{2}+I_{\pi N}^{(p)}\left(m_{N}^{2}\right)-\frac{1}{2} I_{N N}(t)+\frac{1}{32 \pi^{2}}\right\} .
\end{aligned}
$$

APPENDIX B: CONTRIBUTIONS TO THE DIRAC AND PAULI FORM FACTORS

The unrenormalized expressions for the Dirac form factor $F_{1}$ read

$$
\begin{gathered}
F_{1}^{1+3}=\frac{1+\tau_{3}}{2}-\left(\tau_{3} d_{6}+2 d_{7}\right) t \\
F_{1}^{5}=\frac{\stackrel{\circ}{A}^{2}}{8 F^{2}}\left(3-\tau_{3}\right)\left\{I_{\pi}+4 m_{N}^{2} I_{\pi N}^{(p)}\left(m_{N}^{2}\right)-4 m_{N}^{2}\left[M^{2} I_{\pi N N}(t)+I_{N N}(t)\right]\right. \\
\left.+8 m_{N}^{2} I_{\pi N N}^{(00)}(t)+32 m_{N}^{4} I_{\pi N N}^{(P P)}(t)\right\} \\
F_{1}^{6}=-\frac{\tau_{3}}{2 F^{2}} I_{\pi},
\end{gathered}
$$




$$
\begin{gathered}
F_{1}^{7}=\frac{{\stackrel{\circ}{g_{A}}}^{2}}{F^{2}} \tau_{3}\left[I_{\pi}+2 m_{N}^{2} I_{\pi N}^{(p)}\left(m_{N}^{2}\right)\right] \\
F_{1}^{8}=-\frac{\stackrel{\circ}{A}^{2}}{F^{2}} \tau_{3}\left[t I_{\pi \pi}^{(00)}(t)+4 m_{N}^{2} I_{\pi \pi N}^{(00)}(t)+16 m_{N}^{4} I_{\pi \pi N}^{(P P)}(t)\right] \\
F_{1}^{9}=\frac{\tau_{3}}{F^{2}} t I_{\pi \pi}^{(00)}(t) \\
F_{1}^{10}=\frac{2 m_{N}^{3} \stackrel{\circ}{A}^{2}}{F^{2}}\left(3 c_{7}-2 c_{6} \tau_{3}\right) t I_{\pi N N}^{(P P)}(t) \\
F_{1}^{11}=\frac{3 M^{2}}{4 m_{N} F^{2}}\left(1+\tau_{3}\right) c_{2}\left(I_{\pi}-\frac{M^{2}}{32 \pi^{2}}\right)=\mathcal{O}\left(q^{4}\right) .
\end{gathered}
$$

The contributions to the Pauli form factor $F_{2}$ are given by

$$
\begin{gathered}
F_{2}^{2+3+4}=2 m_{N} \tau_{3} c_{6}+m_{N} c_{7}+\left(\tau_{3} d_{6}+2 d_{7}\right) t+2 m_{N}\left(2 e_{54}+\tau_{3} e_{74}\right) t \\
-8 m_{N} M^{2}\left(2 e_{105}+\tau_{3} e_{106}\right), \\
F_{2}^{5}=-\frac{\stackrel{\circ}{A}^{2}}{F^{2}}\left(3-\tau_{3}\right) 4 m_{N}^{4} I_{\pi N N}^{(P P)}(t), \\
F_{2}^{8}=\frac{\stackrel{\circ}{A}^{2}}{F^{2}} \tau_{3} 16 m_{N}^{4} I_{\pi \pi N}^{(P P)}(t), \\
F_{2}^{10}=-\frac{g_{A}^{2} m_{N}}{4 F^{2}}\left(3 c_{7}-2 c_{6} \tau_{3}\right)\left\{I_{\pi}+4 m_{N}^{2} I_{\pi N}^{(p)}\left(m_{N}^{2}\right)+4 m_{N}^{2} M^{2} I_{\pi N N}(t)\right. \\
\left.+4 m_{N}^{2} I_{N N}(t)-16 m_{N}^{2} I_{\pi N N}^{(00)}(t)+8 m_{N}^{2} t\left[I_{\pi N N}^{(P P)}(t)-I_{\pi N N}^{(q q)}(t)\right]\right\}, \\
F_{2}^{11}=-\frac{3 M^{2}}{4 m_{N} F^{2}}\left(1+\tau_{3}\right) c_{2}\left(I_{\pi}-\frac{M^{2}}{32 \pi^{2}}\right)-2 \frac{m_{N}}{F^{2}} \tau_{3} c_{6} I_{\pi}, \\
F_{2}^{12}=\frac{4 m_{N}}{F^{2}} \tau_{3} c_{4} t I_{\pi \pi}^{(00)}(t) .
\end{gathered}
$$

The upper indices refer to the corresponding diagrams of Fig. 11. Note that we did not distinguish between $m$ and $m_{N}$, because the difference only shows up at higher orders. Our results agree with the expressions of Ref. 33] up to terms vanishing in infrared regularization.

[1] S. Weinberg, Physica A 96, 327 (1979).

[2] J. Gasser and H. Leutwyler, Annals Phys. 158, 142 (1984).

[3] S. Scherer, in Advances in Nuclear Physics, Vol. 27, edited by J. W. Negele and E. W. Vogt (Kluwer Academic/Plenum Publishers, New York, 2003).

[4] J. Gasser, M. E. Sainio, and A. Švarc, Nucl. Phys. B307, 779 (1988).

[5] E. Jenkins and A. V. Manohar, Phys. Lett. B 255, 558 (1991); ibid. 259, 353 (1991).

[6] V. Bernard, N. Kaiser, J. Kambor, and U.-G. Meißner, Nucl. Phys. B388, 315 (1992).

[7] V. Bernard, N. Kaiser, and U.-G. Meißner, Nucl. Phys. A611, 429 (1996). 
[8] G. Ecker and M. Mojžiš, Phys. Lett. B 365, 312 (1996).

[9] N. Fettes, U.-G. Meißner, M. Mojžiš, and S. Steininger, Annals Phys. 283, 273 (2000); ibid. 288, 249 (2001).

[10] H. Tang, hep-ph/9607436.

[11] P. J. Ellis and H. B. Tang, Phys. Rev. C 57, 3356 (1998).

[12] T. Becher and H. Leutwyler, Eur. Phys. J. C 9, 643 (1999).

[13] J. Gegelia and G. Japaridze, Phys. Rev. D 60, 114038 (1999).

[14] J. Gegelia, G. Japaridze, and X. Q. Wang, hep-ph/9910260.

[15] M. Lutz, Nucl. Phys. A677, 241 (2000).

[16] M. F. Lutz and E. E. Kolomeitsev, Nucl. Phys. A700, 193 (2002).

[17] T. Fuchs, J. Gegelia, G. Japaridze, and S. Scherer, hep-ph/0302117.

[18] F. J. Ernst, R. G. Sachs, and K. C. Wali, Phys. Rev. 119, 1105 (1960).

[19] R. G. Sachs, Phys. Rev. 126, 2256 (1962).

[20] J. J. Kelly, Phys. Rev. C 66, 065203 (2002).

[21] J. Friedrich and Th. Walcher, hep-ph/0303054.

[22] F. Iachello, A. D. Jackson, and A. Lande, Phys. Lett. B 43, 191 (1973).

[23] P. Kroll, M. Schurmann, and W. Schweiger, Z. Phys. A 338, 339 (1991).

[24] X. D. Ji, Phys. Lett. B 254, 456 (1991).

[25] F. Schlumpf, J. Phys. G 20, 237 (1994).

[26] F. Cardarelli, E. Pace, G. Salme, and S. Simula, Phys. Lett. B 357, 267 (1995).

[27] C. V. Christov, A. Z. Gorski, K. Goeke, and P. V. Pobylitsa, Nucl. Phys. A592, 513 (1995).

[28] P. Mergell, U.-G. Meißner, and D. Drechsel, Nucl. Phys. A596, 367 (1996).

[29] D. H. Lu, A. W. Thomas, and A. G. Williams, Phys. Rev. C 57, 2628 (1998).

[30] S. Boffi, L. Y. Glozman, W. Klink, W. Plessas, M. Radici, and R. F. Wagenbrunn, Eur. Phys. J. A 14, 17 (2002).

[31] H. W. Fearing, R. Lewis, N. Mobed, and S. Scherer, Phys. Rev. D 56, 1783 (1997).

[32] V. Bernard, H. W. Fearing, T. R. Hemmert, and U.-G. Meißner, Nucl. Phys. A635, 121 (1998); ibid. A642, 563 (1998).

[33] B. Kubis and U.-G. Meißner, Nucl. Phys. A679, 698 (2001).

[34] T. Ebertshäuser, H. W. Fearing, and S. Scherer, Phys. Rev. D 65, 054033 (2002).

[35] G. Colangelo, J. Gasser, and H. Leutwyler, Phys. Rev. Lett. 86, 5008 (2001).

[36] S. Scherer, A. Y. Korchin, and J. H. Koch, Phys. Rev. C 54, 904 (1996).

[37] T. Becher and H. Leutwyler, JHEP 0106, 017 (2001).

[38] R. Koch, Nucl. Phys. A448, 707 (1986).

[39] L. E. Price, J. R. Dunning, M. Goitein, K. Hanson, T. Kirk, and R. Wilson, Phys. Rev. D 4, 45 (1971).

[40] C. Berger, V. Burkert, G. Knop, B. Langenbeck, and K. Rith, Phys. Lett. B 35, 87 (1971).

[41] K. M. Hanson, J. R. Dunning, M. Goitein, T. Kirk, L. E. Price, and R. Wilson, Phys. Rev. D 8, 753 (1973).

[42] G. G. Simon, C. Schmitt, F. Borkowski, and V. H. Walther, Nucl. Phys. A333, 381 (1980).

[43] T. Eden et al., Phys. Rev. C 50, 1749 (1994).

[44] I. Passchier et al., Phys. Rev. Lett. 82, 4988 (1999).

[45] M. Ostrick et al., Phys. Rev. Lett. 83, 276 (1999).

[46] C. Herberg et al., Eur. Phys. J. A 5, 131 (1999).

[47] J. Becker et al., Eur. Phys. J. A 6, 329 (1999).

[48] T. Janssens, R. Hofstadter, E. B. Hughes, and M. R. Yearian, Phys. Rev. 142, 922 (1966). 
[49] G. Höhler, E. Pietarinen, I. Sabba Stefanescu, F. Borkowski, G. G. Simon, V. H. Walther, and R. D. Wendling, Nucl. Phys. B114, 505 (1976).

[50] P. Markowitz et al., Phys. Rev. C 48, 5 (1993).

[51] H. Anklin et al., Phys. Lett. B 336, 313 (1994).

[52] E. E. Bruins et al., Phys. Rev. Lett. 75, 21 (1995).

[53] H. Anklin et al., Phys. Lett. B 428, 248 (1998).

[54] W. Xu et al., Phys. Rev. Lett. 85, 2900 (2000).

[55] G. Kubon et al., Phys. Lett. B 524, 26 (2002).

[56] T. Fuchs, J. Gegelia, and S. Scherer, in preparation.

[57] G. Passarino and M. J. Veltman, Nucl. Phys. B160, 151 (1979). 


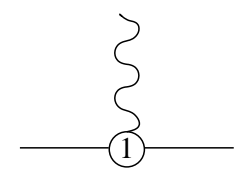

(1)

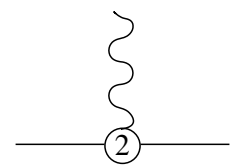

(2)

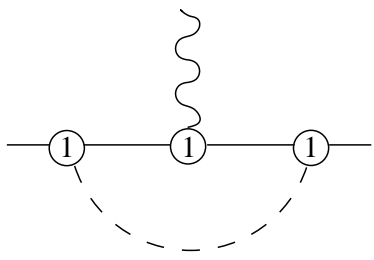

(5)
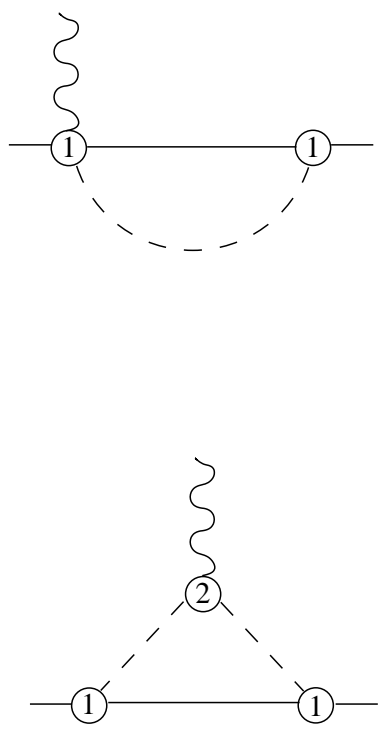

(8)

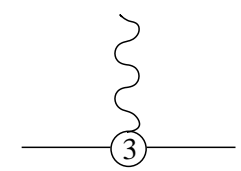

(3)

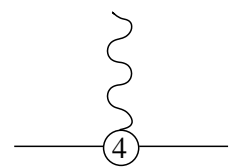

(4)

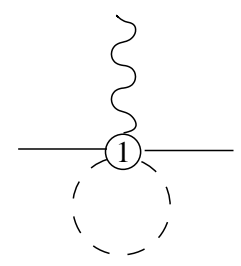

(6)

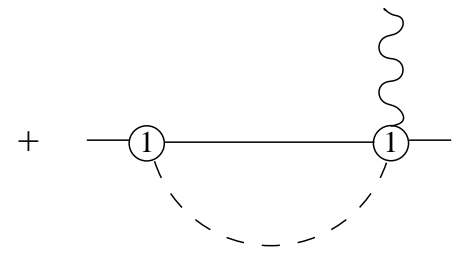

(7)

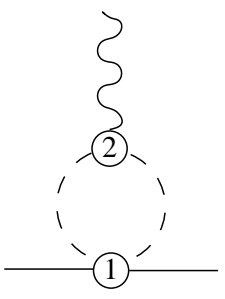

(9)

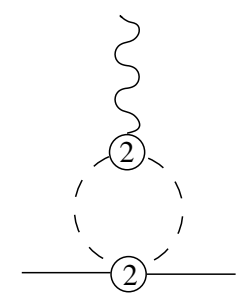

(12)

FIG. 1: Feynman diagrams contributing to the electromagnetic form factors up to and including $\mathcal{O}\left(q^{4}\right)$. External-leg corrections are not shown. Solid, dashed, and wiggly lines refer to nucleons, pions, and photons, respectively. 

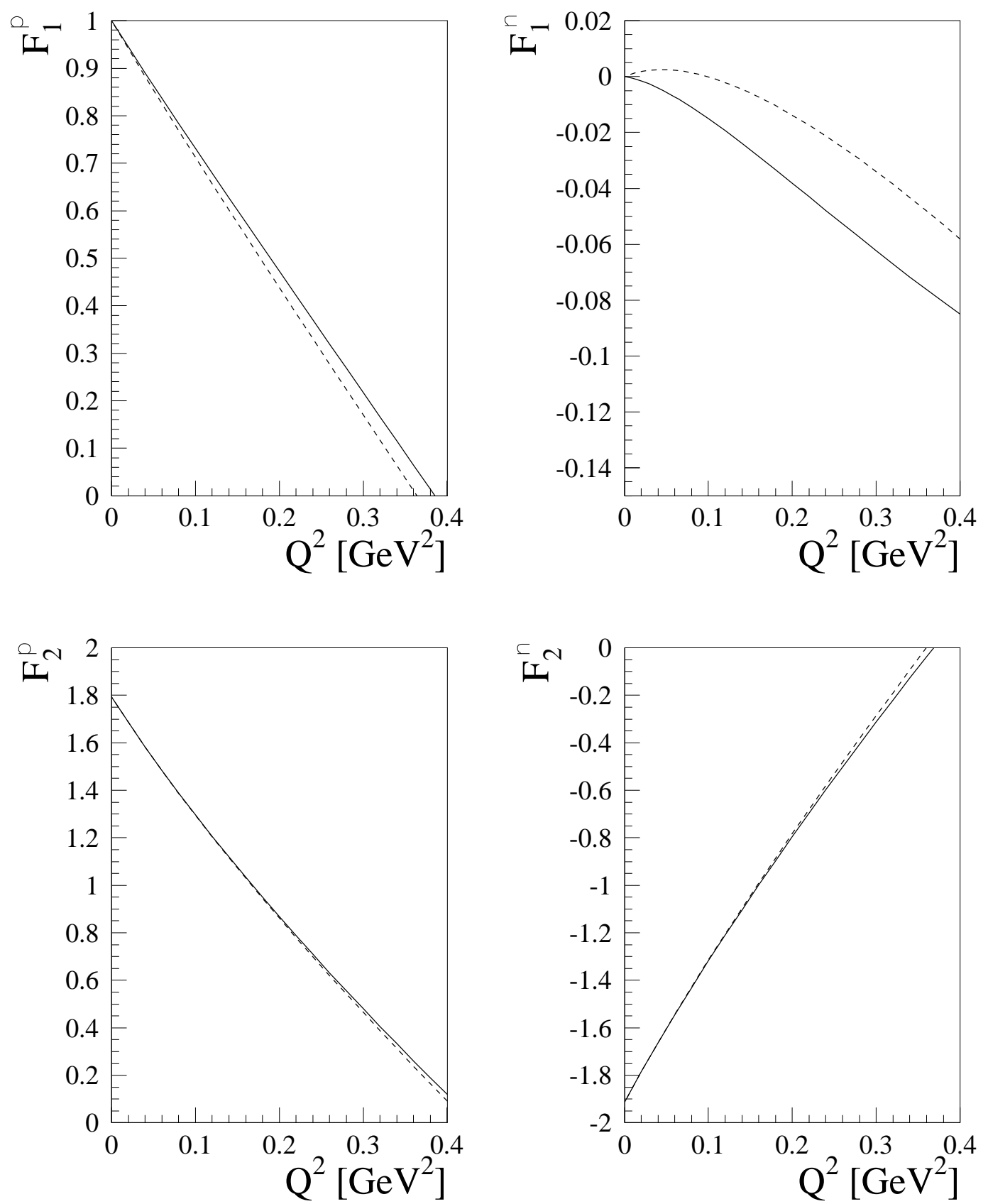

FIG. 2: The Dirac and Pauli form factors of the nucleon in relativistic chiral perturbation theory at $\mathcal{O}\left(q^{4}\right)$. Full lines: our results in the extended on-mass shell (EOMS) scheme; dashed lines: infrared regularization result [33]. 


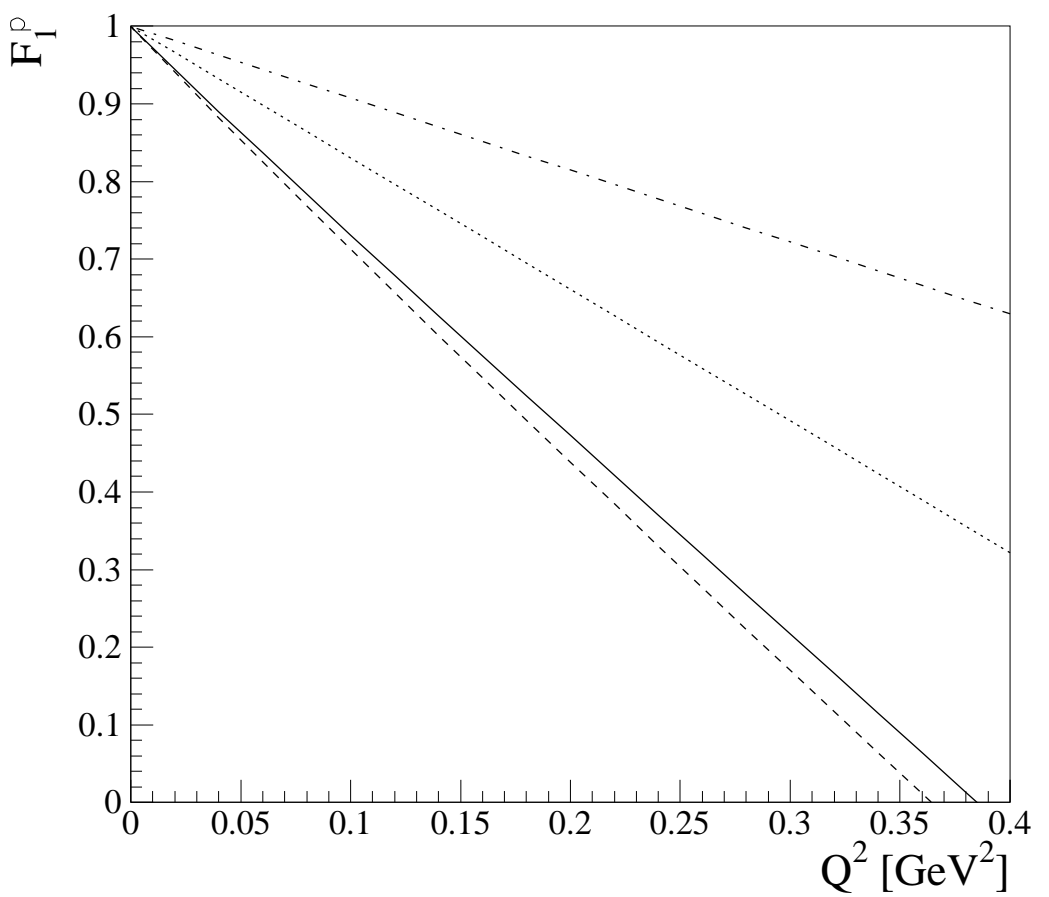

FIG. 3: The Dirac form factor of the proton at $\mathcal{O}\left(q^{4}\right)$. Solid line: our result; dashed line: infrared regularization; dotted line: our result without loop contribution; dashed-dotted line: infraredregularization result without loop contribution. 

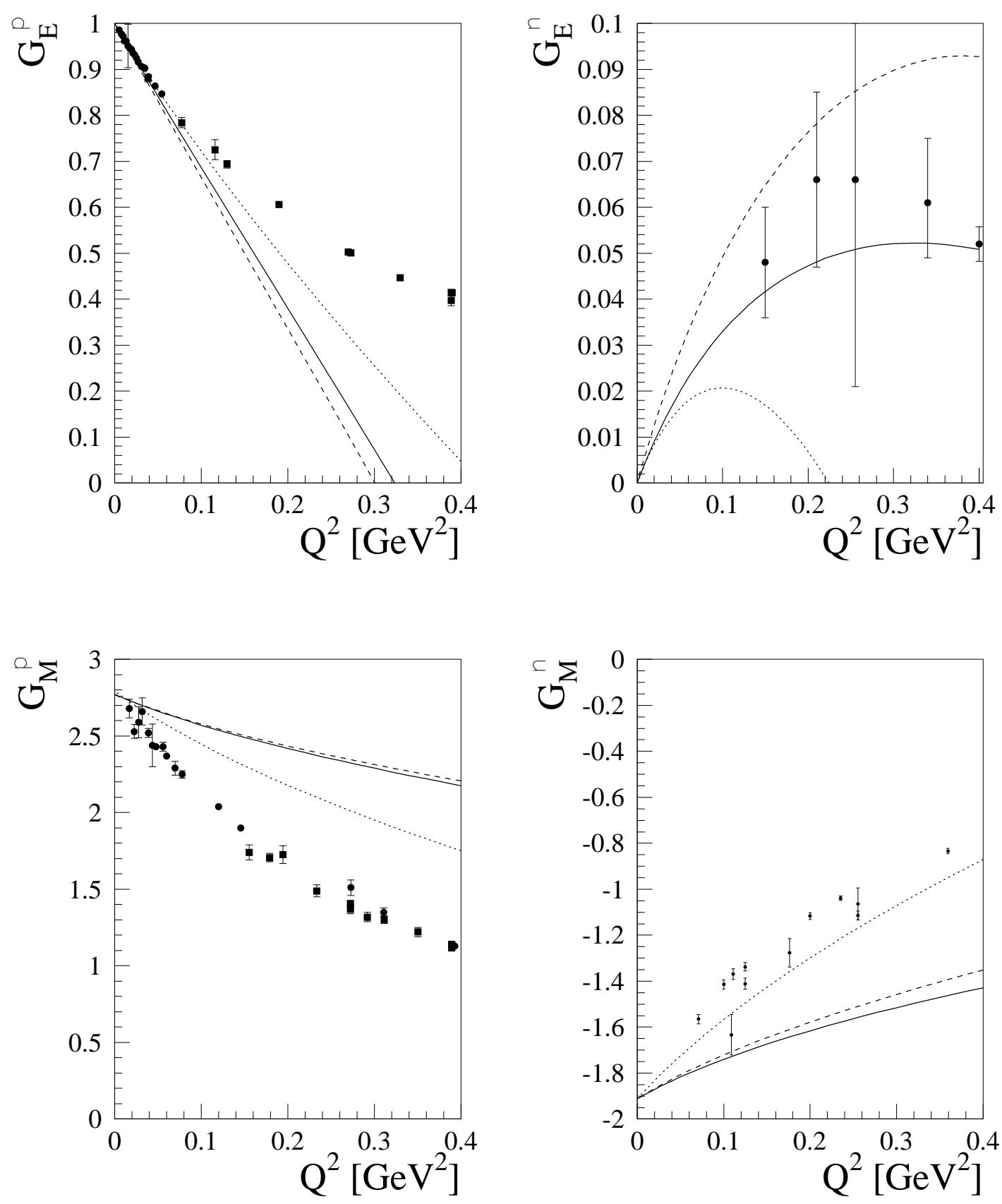

FIG. 4: The Sachs form factors of the nucleon at $\mathcal{O}\left(q^{3}\right)$. The solid, dashed, and dotted lines refer to the results in the EOMS scheme, the infrared regularization 33], and $\mathrm{HB} \chi \mathrm{PT}$ 6, 31], respectively. The experimental data for $G_{\underline{\underline{E}}}^{p}, G_{E}^{n}, G_{M}^{p}$, and $G_{M}^{n}$ are taken from Refs. 39, 40, 41, 42], [43, 44, 45, 46, 47], [40, 41, 48, 49], and [50, 51, 52, 53, 54, 55], respectively. 

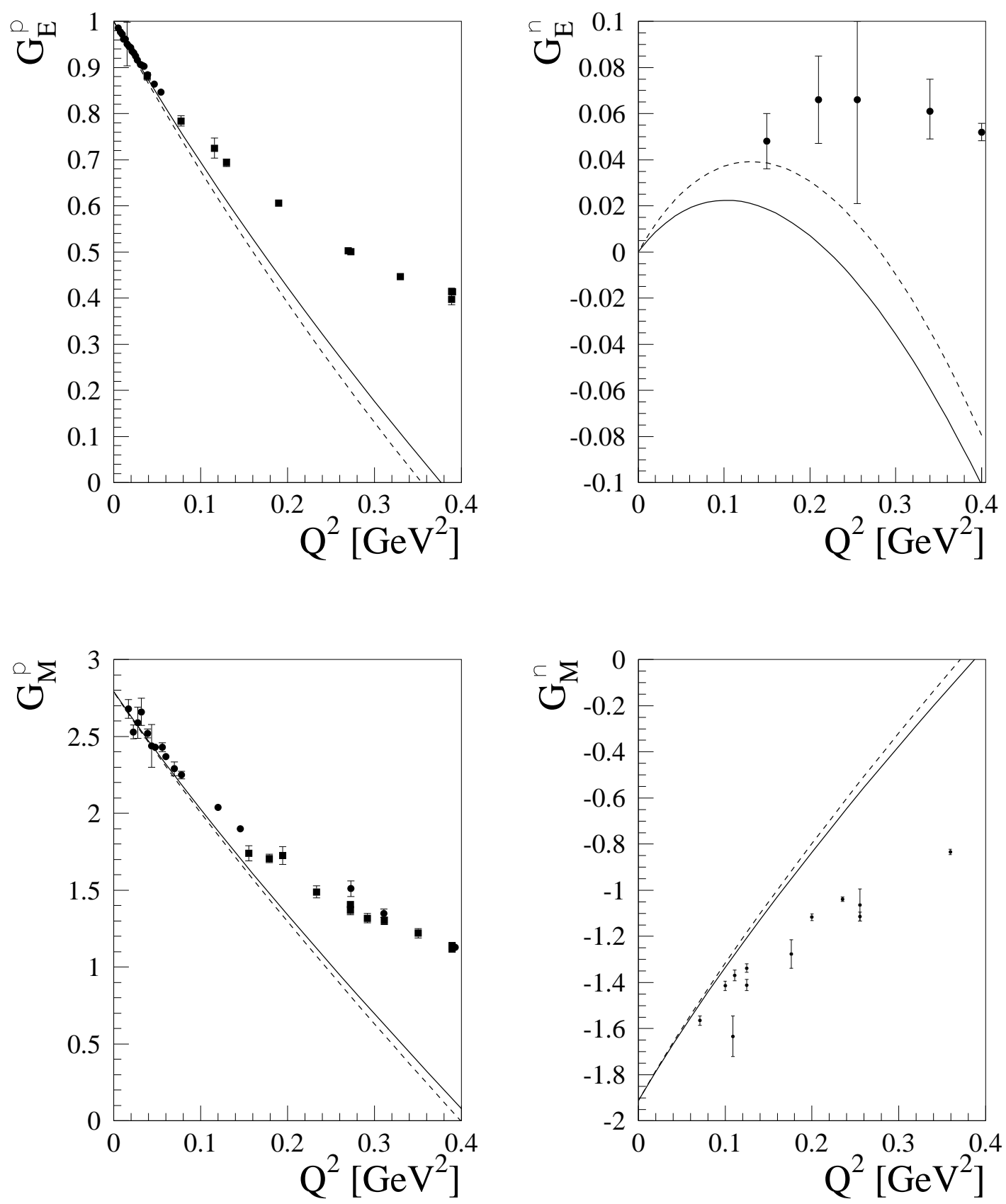

FIG. 5: The Sachs form factors of the nucleon at $\mathcal{O}\left(q^{4}\right)$. The solid and dashed lines refer to the results in the EOMS scheme and the infrared regularization 33], respectively. The experimental data for $G_{E}^{p}, G_{E}^{n}, G_{M}^{p}$, and $G_{M}^{n}$ are taken from Refs. 39, 40, 41, 42], 43, 44, 45, 46, 47], 40, 41, 48, 49], and [50, 51, 52, 53, 54, 55], respectively. 


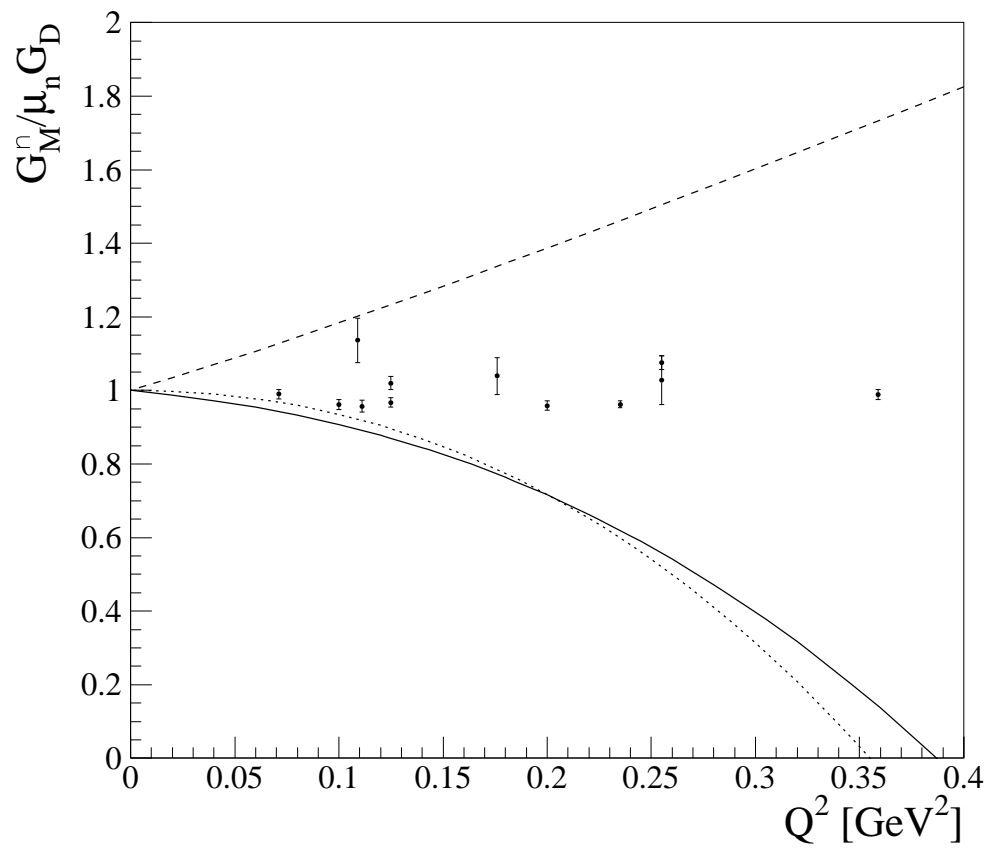

FIG. 6: The magnetic form factor of the neutron divided by $\mu_{n} G_{D}$. The solid and dashed lines refer to the results in the EOMS scheme at $\mathcal{O}\left(q^{4}\right)$ and $\mathcal{O}\left(q^{3}\right)$, respectively. The dotted line is the linear approximation of the dipole form factor, i.e., $\left[1-2 Q^{2} /\left(0.71 \mathrm{GeV}^{2}\right)\right] / G_{D}\left(Q^{2}\right)$. The data are taken from Refs. [50, 51, 52, 53, 54, 55. 


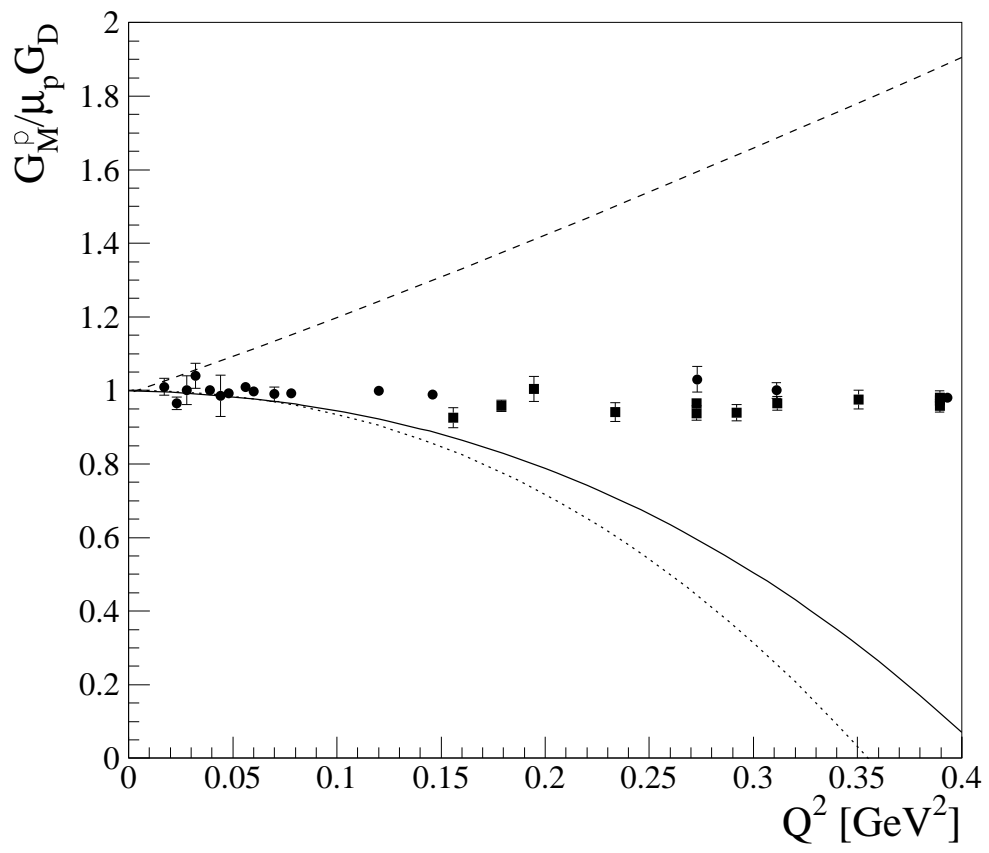

FIG. 7: The magnetic form factor of the proton divided by $\mu_{p} G_{D}$. The solid and dashed lines refer to the results in the EOMS scheme at $\mathcal{O}\left(q^{4}\right)$ and $\mathcal{O}\left(q^{3}\right)$, respectively. The dotted line is the linear approximation of the dipole form factor, i.e., $\left[1-2 Q^{2} /\left(0.71 \mathrm{GeV}^{2}\right)\right] / G_{D}\left(Q^{2}\right)$. The data are taken from Refs. [40, 41, 48, 49]. 


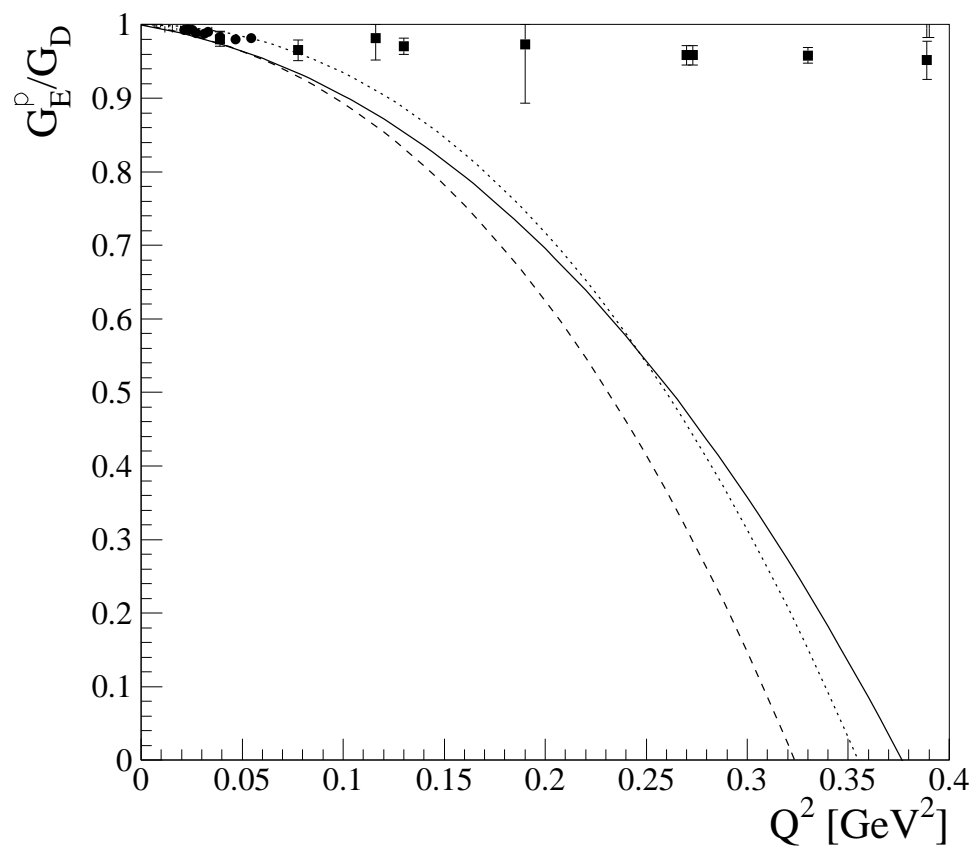

FIG. 8: The electric form factor of the proton divided by $G_{D}$. The solid and dashed lines refer to the results in the EOMS scheme at $\mathcal{O}\left(q^{4}\right)$ and $\mathcal{O}\left(q^{3}\right)$, respectively. The dotted line is the linear approximation of the dipole form factor, i.e., $\left[1-2 Q^{2} /\left(0.71 \mathrm{GeV}^{2}\right)\right] / G_{D}\left(Q^{2}\right)$. The data are taken from Refs. 39, 40, 41, 42]. 